\title{
Effectiveness of Posterolateral Lumbar Fusion Varies with the Physical Properties of Demineralized Bone Matrix Strip
}

\author{
Yong-Soo Choi ${ }^{1}$, Dae-Hee Kim ${ }^{1}$, Ji-Hun Park ${ }^{1}$, Brian Johnstone ${ }^{2}$, Jung-U Yoo ${ }^{2}$ \\ ${ }^{1}$ Department of Orthopaedics, Kwangju Christian Hospital, Gwangju, Korea \\ ${ }^{2}$ Department of Orthopaedics, Oregon Health and Science University, Portland, OR, USA
}

\begin{abstract}
Study Design: A randomized, controlled animal study.
Purpose: To investigate the effectiveness of fusion and new bone formation induced by demineralized bone matrix (DBM) strips with jelly strengths.

Overview of Literature: The form of the DBM can make a difference to the outcome. The effect of different jelly strengths on the ability of DBM to form new bone is not known.

Methods: Forty-eight rabbits were randomized into a control group and two experimental groups. In the control group (group 1), $1.4 \mathrm{~g}$ of autologous iliac crest bone was placed bilaterally. In the experimental groups, a high jelly strength DBM-hyaluronic acid (HA)-gelatin strip (group 2) and a low jelly strength DBM-HA-gelatin strip (group 3) were used. The fusion was assessed with manual manipulation and radiographs. The volume of the fusion mass was determined from computed tomographic images.

Results: The fusion rates as determined by manual palpation were $37.5 \%, 93.8 \%$ and $50.0 \%$ in group 1, group 2, and group 3, respectively $(p<0.05)$. By radiography, the fusion rate of High jelly strength DBM strip was statistically significantly greater than that of the other alternatives $(p<0.05)$. The mean bone volume of the fusion mass as determined by computed tomography was $2,142.2 \pm 318.5$ $\mathrm{mm}^{3}, 3,132.9 \pm 632.1 \mathrm{~mm}^{3}$, and $2,741.5 \pm 380.4 \mathrm{~mm}^{3}$ in group 1 , group 2 , and group 3 , respectively $(p<0.05)$.

Conclusions: These results indicate that differences in the structural and mechanical properties of gelatin that are associated with jelly strength influenced cellular responses such as cell viability and bony tissue ingrowth, facilitating greater bone fusion around high jelly strength implants.
\end{abstract}

Keywords: Demineralized bone matrix; Spinal fusion

\section{Introduction}

Autogenous iliac crest bone graft has been the "gold standard" for spinal fusion. However, morbidity associated with bone graft harvest has led surgeons to seek other potential adjuncts, including bone morphogenetic proteins, demineralized bone matrix (DBM), and graft expanders such as synthetic bone graft and allograft.

DBM is already being used to augment spinal fusion. It has potential advantages over other bone substitutes, including lower immunogenicity than mineralized allograft bone, and the exposure of osteoconductive extracellular

Received Sep 9, 2014; Revised Nov 1, 2014; Accepted Nov 25, 2014

Corresponding author: Yong-Soo Choi

Department of Orthopedic Surgery, Kwangju Christian Hospital,

37 Yangnim-ro, Nam-gu, Gwangju 503-715, Korea

Tel: +82-62-650-5064, Fax: +82-62-650-5066, E-mail: stemcellchoi@gmail.com 
matrix and osteoinductive proteins [1,2]. In previous studies on DBM in spinal fusion, we and other authors found that DBM increased bone formation and facilitated successful posterolateral spinal fusion [3-6].

The wide variability of osteoinductive activity of DBMs is likely to be influenced by the donor, the carrier, and the assorted demineralization and sterilization methods used. A variety of DBMs are available in different forms, such as powders, putties, gels, and sheets, for spinal fusion. The form of the DBM can make a difference to the outcome. For example, Martin et al. [7] reported that DBM sheet and putty forms have a greater capacity to form bone than the DBM gel form in a rabbit model. We have reported that DBM in the form of a strip was able to function as both a bone graft enhancer and a bone graft substitute in posterolateral spinal fusion in an established rabbit model [8]. The formulation consisted of allogeneic DBMs with hyaluronan and gelatin. Gelatin is reported to be an excellent substrate for cell attachment, proliferation, and differentiation. The jelly strength of gelatin can vary with temperature, $\mathrm{pH}$, and gelatin concentration. The effect of different jelly strengths on the ability of DBM to form new bone is not known. The purpose of this study was to investigate the effectiveness of fusion and new bone formation induced by DBM strips with jelly strengths. The study was conducted using the established rabbit model of posterolateral spinal fusion.

\section{Materials and Methods}

\section{Preparation of DBM-hyaluronan (HA)-gelatin strip}

Bilateral femurs and tibiae of 60 sacrificed New Zealand white rabbits (3.5-4 kg, 9-12 months old) were harvested and sent to the Musculoskeletal Transplant Foundation (Edison, NJ, USA), for processing the cortical bone into DBM. The DBM was combined with hyaluronan and porcine gelatin of different jelly strengths to form solid strips. The procedure was identical to that used for processing and production of human DBM, wherein bone harvesting and processing are performed using an aseptic technique.

\section{Control and experimental groups}

Posterolateral spinal fusion was performed between the L5 and L6 lumbar vertebrae in 48 skeletally mature (3-3.5 kg, 7-8 month old) New Zealand White rabbits. The rab- bits were block randomized into three groups: one control group and two experimental groups. In the control group, $1.4 \mathrm{~g}$ of autologous iliac crest bone (group 1) was placed bilaterally. The $1.4 \mathrm{~g}$ of bone was weighed to ensure consistency of implant size and then divided into two equal portions for placement bilaterally. In the experimental groups, a high jelly strength DBM-HA-gelatin strip (group 2) and a low jelly strength DBM-HA-gelatin strip (Group $3)$ were used. The DBM-HA-gelatin strips $(25 \times 10 \times 2 \mathrm{~mm})$ were placed directly over the two transverse processes, bilaterally.

\section{Surgery}

A bilateral intertransverse process posterolateral spinal fusion was performed between L5 and L6, similar to that described in previous published studies [9-11]. A standard posterior midline incision was used, and bone for grafting was obtained through the same surgical incision. Preoperative antibiotics included $0.25 \mathrm{~mL}$ of Dual-cillin (penicillin $\mathrm{G}$ benzathine and penicillin $\mathrm{G}$ procaine injectable aqueous solution 300,000 u/mL, Phoenix Pharmaceuticals Inc., St. Louis, MO, USA) and $0.25 \mathrm{~mL}$ of gentamicin sulfate (Gentocin, $50 \mathrm{mg} / \mathrm{mL}$ gentamicin sulfate, Schering-Plough Animal Health Corp., Kenilworth, NJ, USA). General anesthesia was administered by intramuscular injection of a solution $(0.75 \mathrm{~mL} / \mathrm{kg} / \mathrm{rabbit})$ consisting of $150 \mathrm{mg}$ ketamine hydrochloride (Ketaset III, Fort Dodge Animal Health, Fort Dodge, IA, USA), 30 mg xylazine (AnaSed, Lloyd Laboratories, Shenandoah, IA, USA), and $5 \mathrm{mg}$ acepromazine maleate (Boehringer Ingelheim, Vetmedica Inc., St. Joseph, MO, USA). Bupivacaine hydrochloride $(5 \mathrm{~mL}$ of $0.5 \%$, Abbott Laboratories, North Chicago, IL, USA) was infiltrated intramuscularly into the lumbar paraspinal muscles after a midline skin incision. The transverse processes of L5 and L6 were exposed bilaterally and decorticated with a high-speed carbide burr. Either autologous bone graft or DBM-gel strip was placed over the decorticated transverse processes according to the randomization protocol (Fig. 1). The $1.4 \mathrm{~g}$ of bone was weighed to ensure consistency of implant size and then divided into 2 equal portions for placement bilaterally. The surgical sites were closed with running absorbable sutures after hemostasis was ensured. The rabbits were allowed normal cage activity and they were euthanized at 9 weeks post-surgery by intravenous ear vein injection of phenobarbital (2 mL Fatal-Plus solution, Vortech Phar- 

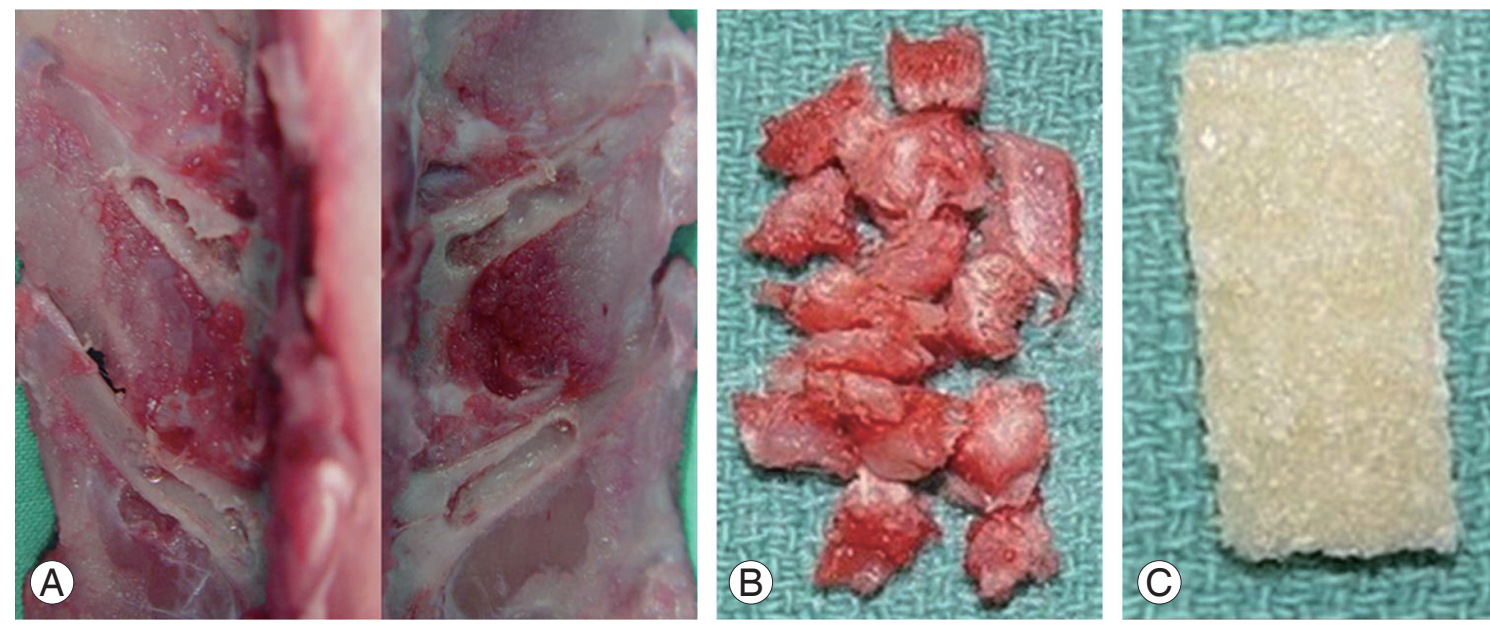

Fig. 1. (A) Graft materials. The transverse processes of $L 5$ and $L 6$ were exposed bilaterally and decorticated with a high-speed carbide burr. (B) Morselized autogenous iliac graft was divided into 2 equal portions, $0.7 \mathrm{~g}$ bilaterally in group 1. (C) Allogeneic demineralized bone matrix containing hyaluronan and gelatin strips $(10 \times 25 \mathrm{~mm}$ each) in groups 2 and 3 .

maceuticals, Dearborn, MI, USA).

\section{Specimen retrieval and manual manipulation test}

After euthanasia, the lumbar vertebrae were dissected with adjacent paravertebral musculature en bloc. A manual manipulation test was performed immediately after harvest, allowing an unencumbered view of the L5-L6 intervertebral disc, and the transverse processes of $\mathrm{L} 5$ and L6 $[12,13]$. A reviewer blinded to the group assignment manipulated the spine in the sagittal plane. Only those specimens with complete absence of motion between the transverse processes of L5 and L6 and at the L5-L6 intervertebral disc were rated as fused spines. The specimens were scored as 0 when gross motion was present, 1 if there was limited motion compared with the adjacent segment, or 2 if absolutely no motion was detected.

\section{Radiographic analysis of fusion}

For radiographic analysis, dorsoventral fine detail radiographs (45 kVps, 1.5 minutes, Faxitron Radiograph Corp., Buffalo Groove, IL, USA) were taken of the specimens. The radiographs were graded in a blinded and independent fashion [9] using a 5-point grading scale.

\section{Volume of bone fusion mass}

The volume of the fusion mass was determined from axial computed tomographic (CT) images of the specimen.
Two-millimeter images with one-mm overlap (scan circle $150 \mathrm{~mm}, 200 \mathrm{Mas}$ ) were produced with a multi-slice spiral CT (Mx 8000 [quad], Marconi Medical Systems, Cleveland, OH, USA). Each axial CT section was digitized using commercially available software (Software MX-view, Marconi Medical Systems), and the fusion mass area was manually delineated with a cursor. The surface area of all cuts encompassing the spinal fusion was then summated and multiplied by the average cut thickness (one mm), yielding a fusion mass volume [9]. An independent evaluator blinded to the block group randomization of the animals determined the fusion mass volume.

\section{Histology}

The spines were frozen at $-20^{\circ} \mathrm{C}$ after manual palpation test and faxitron radiograph. For histologic processing, the spines were subjected to sequential dehydration with ethanol, and then cleared in xylene and embedded in methylmethacrylate. They were cut into sagittal sections approximately $300 \mu \mathrm{m}$ thick using a water-cooled diamond saw (Isomet, Buehler, Lake Bluff, IL, USA). The sections were mounted on 2-mm thick plastic blocks, carefully ordered. Specimens were ground to $100 \mu \mathrm{m}$, polished, and stained with toluidine blue (Fisher Scientific, Fair Lawn, NJ, USA). Selected specimens were subjected to histologic evaluation. Tissues were identified by morphology and by the intensity of the staining to characterize the mineralized tissues. 


\section{Statistics}

Statistical analysis was performed using SigmaStat (Jandel Scientific). The presence of bony fusion in the 2 groups, as judged by manual palpation testing and radiographic scores, was determined using the Fisher exact test. For statistical analysis, radiographic grades 3 and 4 were considered fused. The volume of the bone fusion mass in the groups was also compared using the one-way analysis of variance (ANOVA) (Student-Newman-Keuls method for multiple comparisons).

\section{Results}

Three animals died during surgery due to anesthesiarelated complications before commencement of surgery. Three replacement rabbits were included in the study. All other animals recovered from surgery, and the immediate postoperative period was uneventful. No postoperative wound infections or other complications were observed.

Manual palpation of the excised lumbar spinal segment was used as the first assessment of fusion success. Surgeons blinded to the implant type performed this procedure. The fusion rates as determined by manual palpation were $37.5 \%, 93.8 \%$ and $50.0 \%$ in group 1 , group 2 , and group 3, respectively (Table 1 ). The fusion rate of High jelly strength DBM strip (group 2) was statistically significantly greater than that of the other alternatives $(p<0.005$, group 2 vs. group $1 ; p<0.05$, group 2 vs. group
Table 1. Fusion success as judged by manual palpation

\begin{tabular}{cccc} 
& \multicolumn{3}{c}{ Score } \\
\cline { 2 - 4 } & 0 & 1 & 2 \\
1 & - & 10 & 6 \\
2 & - & 1 & 15 \\
3 & - & 8 & 8 \\
\hline
\end{tabular}

A score 2 was given to those spines that were considered fusion.

Table 2. Radiographic fusion success

\begin{tabular}{ccccc} 
& \multicolumn{4}{c}{ Score } \\
\cline { 2 - 5 } Group & 1 & 2 & 3 & 4 \\
1 & - & 5 & 9 & 2 \\
2 & - & - & 1 & 15 \\
3 & - & 7 & 5 & 4 \\
\hline
\end{tabular}

Scores of 3 (unilateral fusion) and 4 (bilateral fusion) were counted as fusion for statistical analysis.

3; chi-square test). As a second test of fusion success, all spines were subjected to X-ray examination, and surgeons blinded to the implant type scored the radiographs (Fig. 2). In the control group, the fusion rate determined by fine detail radiography was $68.7 \%$ (autograft bone, group 1). In the experimental groups, the fusion rates were $100 \%$ in group 2 and $56.3 \%$ in group 3 (Table 2). The fusion rate of High jelly strength DBM strip (group 2) was statistically significantly greater than that of the other alternatives
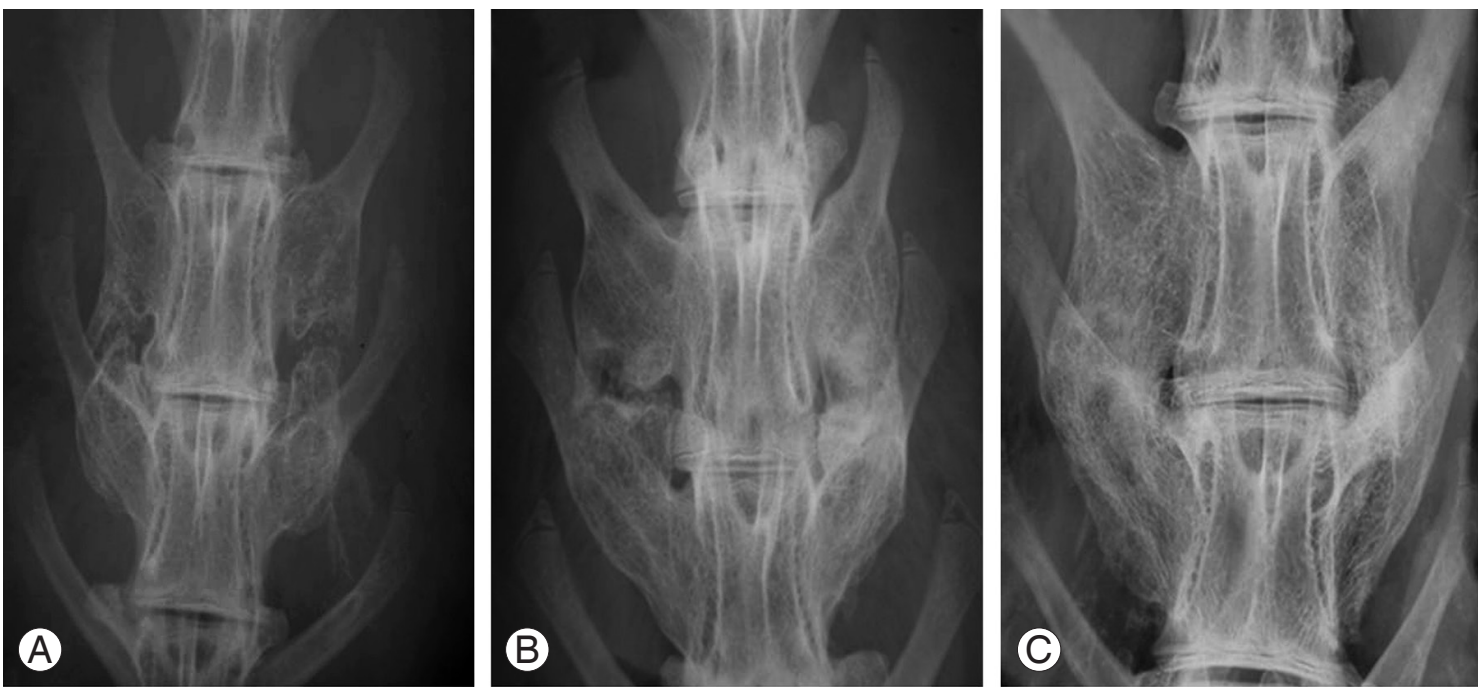

Fig. 2. Radiographic fusion score. (A) Score 2, bone mass present with lucency bilaterally. (B) Score 3, bone mass present bilaterally with lucency on one side. (C) Score 4, bone mass present bilaterally without lucency. 
( $p<0.05$, group 2 vs. group $1 ; p<0.005$, group 2 vs. group 3 ; chi-square test).

The mean bone volume ( \pm standard deviation) of the fusion mass as determined by CT was $2,142.2 \pm 318.5 \mathrm{~mm}^{3}$, $3,132.9 \pm 632.1 \mathrm{~mm}^{3}$, and $2,741.5 \pm 380.4 \mathrm{~mm}^{3}$ in group 1 , group 2, and group 3, respectively. The mineralized bone volume was greater in the DBM-strip groups compared with the autograft bone group (group 2 vs. group 1; group 3 vs. group $1, p<0.05$; ANOVA). The high and low jelly strength DBM strip groups were not statistically different from each other in terms of bone volume (group 2 vs. group 3, $p=0.054$, ANOVA, Student-Newman-Keuls method for multiple comparisons).

Histologically, there was no evidence of chronic or acute inflammation in any of the groups. The quality of bone in the fusion mass was indistinguishable between the groups, with endochondral ossification evident in all groups (Fig. 3A). There was fibrous tissue between new bone in the fusion masses with non-unions (Fig. 3B, C).

\section{Discussion}

A variety of bone graft alternatives have been developed. One approach to optimize an osteoinductive preparation is to focus on isolating or synthesizing individual bone morphogenetic proteins and combining them with suitable carriers for delivery to a skeletal site. Potent osteoinductive growth factors such as bone morphogenetic proteins have demonstrated promising preclinical efficacy results [13-18]. However, in a 2002 review article, Poynton and Lane [19] described the safety issues associated with the use of bone morphogenetic proteins in spine applications, including possible bony overgrowth, interactions with exposed dura, a risk of cancer, local, systemic, and reproductive toxicity, immunogenicity, osteoclastic activation, and effects on distal organs. In 2008, the US Food and Drug Administration issued a Public Health Notification [20] regarding life-threatening complications associated with recombinant human bone morphogenetic protein 2 (rhBMP-2) use; these complications were associated with swelling of the neck and throat tissue resulting in compression of the airway and/or neurological structures. Human demineralized bone matrices with suitable carriers that help improve their handling properties represent an alternative to BMPs, which may be cost effective for routine use.

DBM is widely used as a bone void filler at sites that are intrinsically non-load bearing. Since dry DBM is difficult to deliver and contain at the surgical site, commercially available DBMs are constituted with carriers to improve handling characteristics for surgeons. In most instances, these carriers are inert, non-bone derived viscous substances that help to hold the DBM particles together. The formulation evaluated in this study consisted of allogeneic DBM combined with hyaluronan and gelatin in the form of a strip. Gelatin derived from collagen is reported to be an excellent substrate for cell attachment, proliferation, and differentiation [21-23]. The disadvantages of using gelatin as a scaffold material for tissue repair are its low biomechanical stiffness and rapid biodegradation [22]. However, stability is achieved by the hyaluronan component, which can also facilitate in vivo differentiation of mesenchymal progenitor cells [24]. Hyaluronan/gelatin combined scaffolds have been shown to be effective for formation of skeletal tissues [25]. We reported that a novel formulation of DBM in the form of a strip was able to function as both a bone graft enhancer and a bone graft substitute in posterolateral spinal fusion in an established
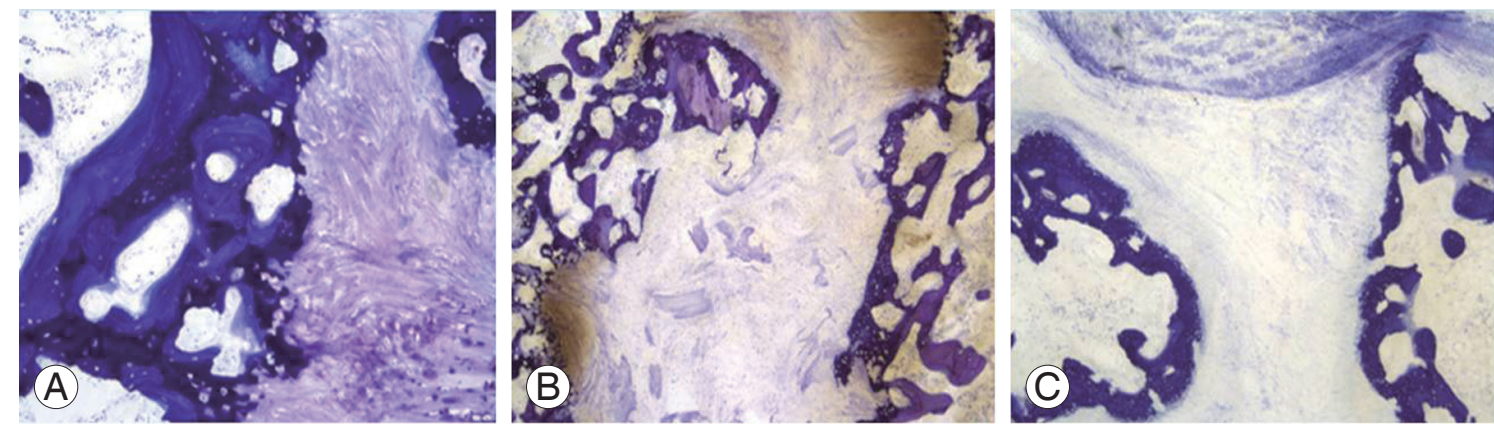

Fig. 3. Histologic findings of each group (toluidine blue stain). Histologic finding showed new bone formation through endochondral ossification in allogeneic demineralized bone matrix strip in groups 2 and $3(\times 10)(A)$. However, there was fibrous tissue between new bone in nonunion cases in group $3(\times 4)(B)$ and group 1 ( $\times 4)(C)$. 
rabbit model [8].

The results of the present study indicate that osteoinductivity of high jelly strength DBM-HA-gelatin strip appears to be greater than that of low jelly strength DBMHA-gelatin Strip as demonstrated by the higher rate of fusion. Gelatin is obtained by thermal, chemical, or physical denaturation of collagen. A lower extraction temperature can produce gelatin with a greater Bloom index, which is a measure of the stiffness of gelatin gels (jelly strength) [26]. It has been reported that an increase in Bloom index of gelatin not only alters the mechanical properties but also leads to a significant reduction in water-absorbing capacity of gelatin membranes [27,28]. Bigi et al. [29] reported that the mechanical properties of gelatin films are greatly affected by the presence of hydroxyapatite and change as a function of inorganic phase content in hydroxyapatite-gelatin films. They studied the influence of the renaturation level of gelatin on the mechanical and swelling properties of gelatin films and concluded that the triple-helix content, calculated from the values of the enthalpy of denaturation, is associated with increases in the Bloom index [27].

\section{Conclusions}

These results indicate that differences in the structural and mechanical properties of gelatin that are associated with jelly strength influenced cellular responses such as cell viability and bony tissue ingrowth, facilitating greater bone fusion around high jelly strength implants.

\section{Conflict of Interest}

No potential conflict of interest relevant to this article was reported.

\section{References}

1. Guizzardi S, Di Silvestre M, Scandroglio R, Ruggeri A, Savini R. Implants of heterologous demineralized bone matrix for induction of posterior spinal fusion in rats. Spine (Phila Pa 1976) 1992;17:701-7.

2. Marinak KW, Mellonig JT, Towle HJ. The osteogenic potential of two human demineralized bone preparations using a xenogeneic model. J Periodontol 1989; 60:12-8.

3. Ragni P, Ala-Mononen P, Lindholm TS. Spinal fusion induced by porous hydroxyapatite blocks (HA): experimental comparative study with HA, demineralized bone matrix and autogenous bone marrow. Ital J Orthop Traumatol 1993;19:133-44.

4. Ragni P, Lindholm TS. Interaction of allogeneic demineralized bone matrix and porous hydroxyapatite bioceramics in lumbar interbody fusion in rabbits. Clin Orthop Relat Res 1991;(272):292-9.

5. Frenkel SR, Moskovich R, Spivak J, Zhang ZH, Prewett AB. Demineralized bone matrix. Enhancement of spinal fusion. Spine (Phila Pa 1976) 1993;18: 1634-9.

6. Sassard WR, Eidman DK, Gray PM, et al. Augmenting local bone with Grafton demineralized bone matrix for posterolateral lumbar spine fusion: avoiding second site autologous bone harvest. Orthopedics 2000;23:1059-64.

7. Martin GJ Jr, Boden SD, Titus L, Scarborough NL. New formulations of demineralized bone matrix as a more effective graft alternative in experimental posterolateral lumbar spine arthrodesis. Spine (Phila Pa 1976) 1999;24:637-45.

8. Choi Y, Oldenburg FP, Sage L, Johnstone B, Yoo JU. A bridging demineralized bone implant facilitates posterolateral lumbar fusion in New Zealand white rabbits. Spine (Phila Pa 1976) 2007;32:36-41.

9. Curylo LJ, Johnstone B, Petersilge CA, Janicki JA, Yoo JU. Augmentation of spinal arthrodesis with autologous bone marrow in a rabbit posterolateral spine fusion model. Spine (Phila Pa 1976) 1999;24:434-8.

10. Boden SD, Schimandle JH, Hutton WC. An experimental lumbar intertransverse process spinal fusion model: radiographic, histologic, and biomechanical healing characteristics. Spine (Phila Pa 1976) 1995; 20:412-20.

11. Boden SD, Schimandle JH, Hutton WC. Lumbar intertransverse-process spinal arthrodesis with use of a bovine bone-derived osteoinductive protein: a preliminary report. J Bone Joint Surg Am 1995;77:140417.

12. Boden SD, Martin GJ Jr, Morone M, Ugbo JL, Titus L, Hutton WC. The use of coralline hydroxyapatite with bone marrow, autogenous bone graft, or osteoinductive bone protein extract for posterolateral lumbar spine fusion. Spine (Phila Pa 1976) 1999;24:320-7.

13. Boden SD, Martin GJ Jr, Morone MA, Ugbo JL, Moskovitz PA. Posterolateral lumbar intertransverse pro- 
cess spine arthrodesis with recombinant human bone morphogenetic protein 2 /hydroxyapatite-tricalcium phosphate after laminectomy in the nonhuman primate. Spine (Phila Pa 1976) 1999;24:1179-85.

14. Boden SD, Zdeblick TA, Sandhu HS, Heim SE. The use of rhBMP-2 in interbody fusion cages: definitive evidence of osteoinduction in humans: a preliminary report. Spine (Phila Pa 1976) 2000;25:376-81.

15. Grauer JN, Patel TC, Erulkar JS, Troiano NW, Panjabi MM, Friedlaender GE. 2000 Young Investigator Research Award winner: evaluation of OP-1 as a graft substitute for intertransverse process lumbar fusion. Spine (Phila Pa 1976) 2001;26:127-33.

16. Martin GJ Jr, Boden SD, Marone MA, Marone MA, Moskovitz PA. Posterolateral intertransverse process spinal arthrodesis with rhBMP-2 in a nonhuman primate: important lessons learned regarding dose, carrier, and safety. J Spinal Disord 1999;12:179-86.

17. Sandhu HS, Khan SN, Suh DY, Boden SD. Demineralized bone matrix, bone morphogenetic proteins, and animal models of spine fusion: an overview. Eur Spine J 2001;10 Suppl 2:S122-31.

18. Viggeswarapu M, Boden SD, Liu Y, et al. Adenoviral delivery of LIM mineralization protein-1 induces new-bone formation in vitro and in vivo. J Bone Joint Surg Am 2001;83:364-76.

19. Poynton AR, Lane JM. Safety profile for the clinical use of bone morphogenetic proteins in the spine. Spine (Phila Pa 1976) 2002;27:S40-8.

20. US Food and Drug Administration. FDA public health notification: life-threatening complications associated with recombinant human bone morphogenetic protein in cervical spine fusion [Internet]. Silver Spring, MD: US Food and Drug Administration; 2013 [cited 2015 Apr 22]. Availavle from: http:// www.fda.gov/MedicalDevices/Safety/AlertsandNo-
tices/PublicHealthNotifications/ucm062000.htm.

21. Hecht BP, Fischgrund JS, Herkowitz HN, Penman L, Toth JM, Shirkhoda A. The use of recombinant human bone morphogenetic protein 2 (rhBMP-2) to promote spinal fusion in a nonhuman primate anterior interbody fusion model. Spine (Phila Pa 1976) 1999;24:629-36.

22. Kito H, Matsuda T. Biocompatible coatings for luminal and outer surfaces of small-caliber artificial grafts. J Biomed Mater Res 1996;30:321-30.

23. Grande DA, Halberstadt C, Naughton G, Schwartz R, Manji R. Evaluation of matrix scaffolds for tissue engineering of articular cartilage grafts. J Biomed Mater Res 1997;34:211-20.

24. Solchaga LA, Dennis JE, Goldberg VM, Caplan AI. Hyaluronic acid-based polymers as cell carriers for tissue-engineered repair of bone and cartilage. J Orthop Res 1999;17:205-13.

25. Angele P, Kujat R, Nerlich M, Yoo J, Goldberg V, Johnstone B. Engineering of osteochondral tissue with bone marrow mesenchymal progenitor cells in a derivatized hyaluronan-gelatin composite sponge. Tissue Eng 1999;5:545-54.

26. Usta M, Piech DL, MacCrone RK, Hillig WB. Behavior and properties of neat and filled gelatins. Biomaterials 2003;24:165-72.

27. Lai JY, Lin PK, Hsiue GH, Cheng HY, Huang SJ, Li YT. Low Bloom strength gelatin as a carrier for potential use in retinal sheet encapsulation and transplantation. Biomacromolecules 2009;10:310-9.

28. Bigi A, Panzavolta S, Rubini K. Relationship between triple-helix content and mechanical properties of gelatin films. Biomaterials 2004;25:5675-80.

29. Bigi A, Panzavolta S, Roveri N. Hydroxyapatite-gelatin films: a structural and mechanical characterization. Biomaterials 1998;19:739-44. 